\title{
On massive MIMO performance with semi-orthogonal pilot-assisted channel estimation
}

\author{
Hua Zhang, Xinru Zheng, Wei Xu* and Xiaohu You
}

\begin{abstract}
With the rapidly increasing demand for high-speed data transmission and a growing number of terminals, massive multiple-input multiple-output (MIMO) has been shown promising to meet the challenges owing to its high spectrum efficiency. Although massive MIMO can efficiently improve the system performance, usage of orthogonal pilots and growing terminals causes large resource consumption especially when the coherence interval is short. This paper proposes a semi-orthogonal pilot design with simultaneous data and pilot transmission. In the proposed technique, we exploit the asymptotic channel orthogonality in massive MIMO systems, with which a successive interference cancellation (SIC)-based channel estimation is applied to mitigate the mutual interference between data and pilot. We derived the theoretical expressions of the achievable rates in massive MIMO systems with our proposed pilot design. Further discussion on performance verifies the superiority of our proposed pilot design for high or low signal-to-noise-ratios (SNRs) with any coherence interval length. And simulation results show that the proposed pilot design can achieve a significant performance improvement with reduced pilot resource consumption compared with the conventional orthogonal pilots.
\end{abstract}

Keywords: Massive MIMO; Multiuser; Semi-orthogonal pilot design; Interference cancellation

\section{Introduction}

With a rapidly increasing demand for high data rates, as well as the growing number of serving users, massive multiple-input multiple-output (MIMO) is emerging as a promising technology to meet the challenge by providing a significant increment in reliability and data rate for wireless communications [1-3].

For MIMO systems, channel state information (CSI) is crucial for achieving multi-antenna gains. It becomes more challenging in massive MIMO systems due to numerous antennas at the base station (BS). Massive MIMO requires a large number of pilots if frequencydivision duplexing (FDD) is used since the burden for downlink pilots is proportional to the number of $\mathrm{BS}$ antennas, while for time-division duplexing (TDD) [3,4], uplink training is an effective method to obtain CSI by exploiting channel reciprocity. Generally, orthogonal pilot

*Correspondence: wxu@seu.edu.cn

National Mobile Communications Research Lab., Southeast University, Nanjing, 210096, China patterns are widely used for multi-channel estimation. It is well understood that the length of orthogonal pilots equals at least the number of users in a cell, which is in general much smaller than the number of massive BS antennas. However, even in TDD, the required resource for orthogonal pilots increases dramatically in a multi-cell massive MIMO system. Moreover, under the restriction of coherence interval duration and increasing user numbers, the same set of orthogonal pilots is reused for adjacent cells, thus pilot contamination [5-7] occurs in a muticell MIMO system. When the BS estimates the channel for a particular user, it may obtain a channel estimate contaminated by adjacent cell users that share the same pilot.

It has been revealed in $[2,8]$ that pilot contamination becomes a bottleneck that limits the performance benefits of massive MIMO. To solve this problem, recent studies [9-11] proposed various approaches tackling with pilot contamination. Although they tried to alleviate the pilot contamination between multiple cells, they still use orthogonal pilots in a single cell, which implies large

\section{Springer}

(c) 2014 Zhang et al:- licensee Springer. This is an Open Access article distributed under the terms of the Creative Commons Attribution License (http://creativecommons.org/licenses/by/4.0), which permits unrestricted use, distribution, and reproduction in any medium, provided the original work is properly credited. 
pilot resource consumption, especially for short coherence interval.

Considering the pilot resource consumption as well as the importance of channel estimation's accuracy, an efficient pilot design is essential for achieving full potential of massive MIMO systems. However, as far as we know, little attention has been paid to pilot design in a massive MIMO system. An exception [12] studied the pilot sequence design which matters little about pilot resource consumption. Therefore, in this work, we study the problem of an efficient pilot design by exploiting the asymptotic channel orthogonality [13] incorporated with successive interference cancellation (SIC) in massive MIMO systems.

The technical contributions of this work are summarized as follows: We present a novel pilot design with low resource consumption. In the proposed technique, we allow simultaneous data and pilot transmission and insert shifted pilot locations in slots, i.e., different users transmit pilots in different slots. It takes advantage of the asymptotic channel orthogonality for massive MIMO. Hence the mutual interference between data and pilot due to a semi-orthogonal pilot design can be mitigated by SIC. Numerical results show that the proposed pilot design outperforms the conventional orthogonal pilots. In particular, for low or high signal-to-noise-ratios (SNRs), we also theoretically prove the superiority of our proposed pilot design.

The paper is organized as follows. In Section 2, we describe the system model and transmission protocol of conventional massive MIMO systems. Section 3 addresses the transmission scheme of massive MIMO systems with the proposed pilot design. We analyze the achievable rates of both the uplink and downlink in Section 4. In Section 5, we deduce the asymptotic achievable rate as the SNR tends to infinity and zero. In Section 6, numerical results show that the proposed pilot design increases data transmission rates in various scenarios. Section 7 contains our conclusions.

\section{System model with orthogonal pilots}

We consider a cellular system composed of one BS with $M$ antennas and $K(K \ll M)$ single-antenna users. Let $\rho_{p}, \rho_{u}$, and $\rho_{d}$ be the pilot SNR, the uplink SNR, and the downlink SNR, respectively. Denote $\mathbf{g}_{k}=\sqrt{\beta_{k}} \mathbf{h}_{k}$ as the channel vector between the BS and the $k$ th user, where $\mathbf{h}_{k}$, the corresponding small-scale fading vector, is i.i.d $\mathcal{C N}\left(\mathbf{0}, \mathbf{I}_{M}\right)$ and $\sqrt{\beta_{k}}$ models the geometric attenuation as well as shadowing effects which is assumed to be constant and known a priori. We assume channel obeys reciprocity in TDD, i.e., the channel factors are the same for both the uplink and downlink, and $\mathbf{h}_{k}$ remains constant during a coherence interval of length $T$.

The transmission scheme of a conventional massive MIMO system with orthogonal pilots is shown in Figure 1, where $\tau_{o p}\left(\tau_{o p} \geq K\right)$ and $D_{o p}$ respectively represent the length of pilot and downlink data. Following our study, the orthogonal pilots is also referred to as the conventional pilot design and as a benchmark. Since $\mathbf{h}_{k}$ changes over coherence intervals, without loss of generality, we take the communication between the BS and users in a coherence interval as an example for deeper analysis. From Figure 1, a coherence interval is organized in three phases: uplink channel estimation period, uplink data transmission period, and downlink data transmission period.

The conventional pilot design in massive MIMO systems utilizing orthogonal pilots can prevent pilot contamination within one cell and obtain relatively accurate channel estimates [2]. However, the required pilot overhead is $\frac{\tau_{o p}}{T}\left(\tau_{o p} \geq K\right)$ for each user in a cell, which is too large especially when $T$ is small and $K$ is large in massive MIMO systems. In the next section, we will propose a possible pilot design which can keep a balance between the efficiency of data transmission and performance of the system.

\section{Semi-orthogonal pilot design}

In this section, we propose a semi-orthogonal pilot design with shifted locations, which reduces the pilot overhead while guaranteeing the system performance due to the merit of asymptotic channel orthogonality in massive MIMO systems.

The transmission scheme with the proposed pilot design is shown in Figure 2, where $\tau_{p p}$ and $D_{p p}$ respectively denote the length of pilot and downlink data, and $q_{1}^{2}[1], q_{1}^{3}[1], q_{1}[1]$ refer to the definitions in Table 1 . In the first coherence interval, when the first user transmits a pilot, the other users are mute so that the BS can estimate the first user's channel without contamination from other users. When the second user transmits a pilot, the first user transmits data while the other users still remain quiet, and so forth. In brief, when one user transmits a pilot, other users whose channels have already been estimated can transmit uplink data. And the BS adopts SIC to decontaminate interference from other users by exploiting the estimated channel information and gets all channel estimates. The channel estimates can be used in the following uplink data reception and downlink data transmission. As for the second coherence interval, all users, except the one who transmits a pilot, can transmit uplink data simultaneously during the channel estimation stage. The BS can utilize the known channel information estimated either from the previous or the present time to remove the contamination from other users.

Remark 1. Note that the pilot overhead for each user in the $i$ th $(i>1)$ coherence interval of the proposed pilot design is $\frac{1}{T}$ from Figure 2. And $\frac{1}{T} \ll \frac{K}{T}$ especially when $T$ is small. As for the first coherence interval of the 


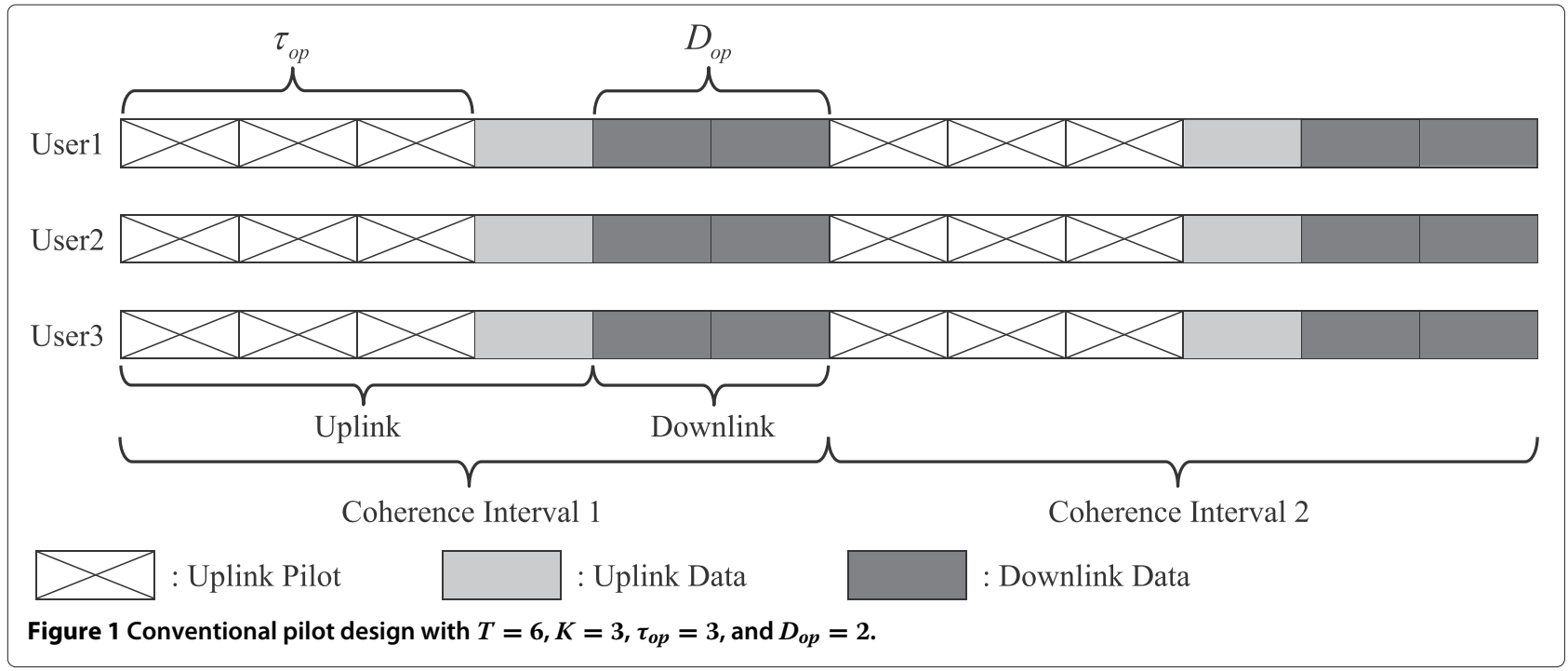

proposed pilot design, the pilot overhead is $\frac{K+1}{2 T}$, which is also smaller than $\frac{K}{T}$ of conventional orthogonal pilots.

Due to the difference between the communications in the first and the $i$ th $(i>1)$ coherence intervals, the uplink and downlink data transmissions as well as the channel estimation are elaborated in detail in the following subsections. Before the elaboration, we first show notational definitions in Table 1, where parameter $i$ represents the $i$ th coherence interval. Besides, we replace $\mathbf{g}_{k}$ with $\mathbf{g}_{k}[i]$ to signify the channel vector between the BS and the $k$ th user.

\subsection{Communication in the first coherence interval}

\subsubsection{Uplink of the first user}

Before data transmission, the first symbol of the first coherence interval is reserved for uplink channel estimation. For the first user in the first coherence interval, it transmits pilot while the other users are mute. The received signal at the $B S$ is

$$
\mathbf{y}^{1}[1]=\sqrt{\rho_{p}} \mathbf{g}_{1}[1] \varphi_{1}+\mathbf{w}^{1}[1] .
$$

Then the BS gets an minimum mean squared error (MMSE) [14,15] estimate:

$$
\hat{\mathbf{g}}_{1}[1]=\frac{\sqrt{\rho_{p}} \beta_{1}}{1+\rho_{p} \beta_{1}} \mathbf{y}^{1}[1] \varphi_{1}^{*} \text {. }
$$

Generally, the channel can be decomposed as $\mathbf{g}_{1}[1]=$ $\tilde{\mathbf{g}}_{1}[1]+\hat{\mathbf{g}}_{1}[1]$. From the properties of MMSE estimation, $\hat{\mathbf{g}}_{1}[1] \sim \mathcal{C N}\left(\mathbf{0}, \sigma_{1,1}^{2} \mathbf{I}_{M}\right), \quad \tilde{\mathbf{g}}_{1}[1] \sim \mathcal{C N}\left(\mathbf{0}, \varepsilon_{1,1}^{2} \mathbf{I}_{M}\right)$ is the independent estimation error, where $\varepsilon_{1,1}^{2}=\beta_{1}-\sigma_{1,1}^{2}$ and $\sigma_{1,1}^{2}=\frac{\rho_{p} \beta_{1}^{2}}{1+\rho_{p} \beta_{1}}$.

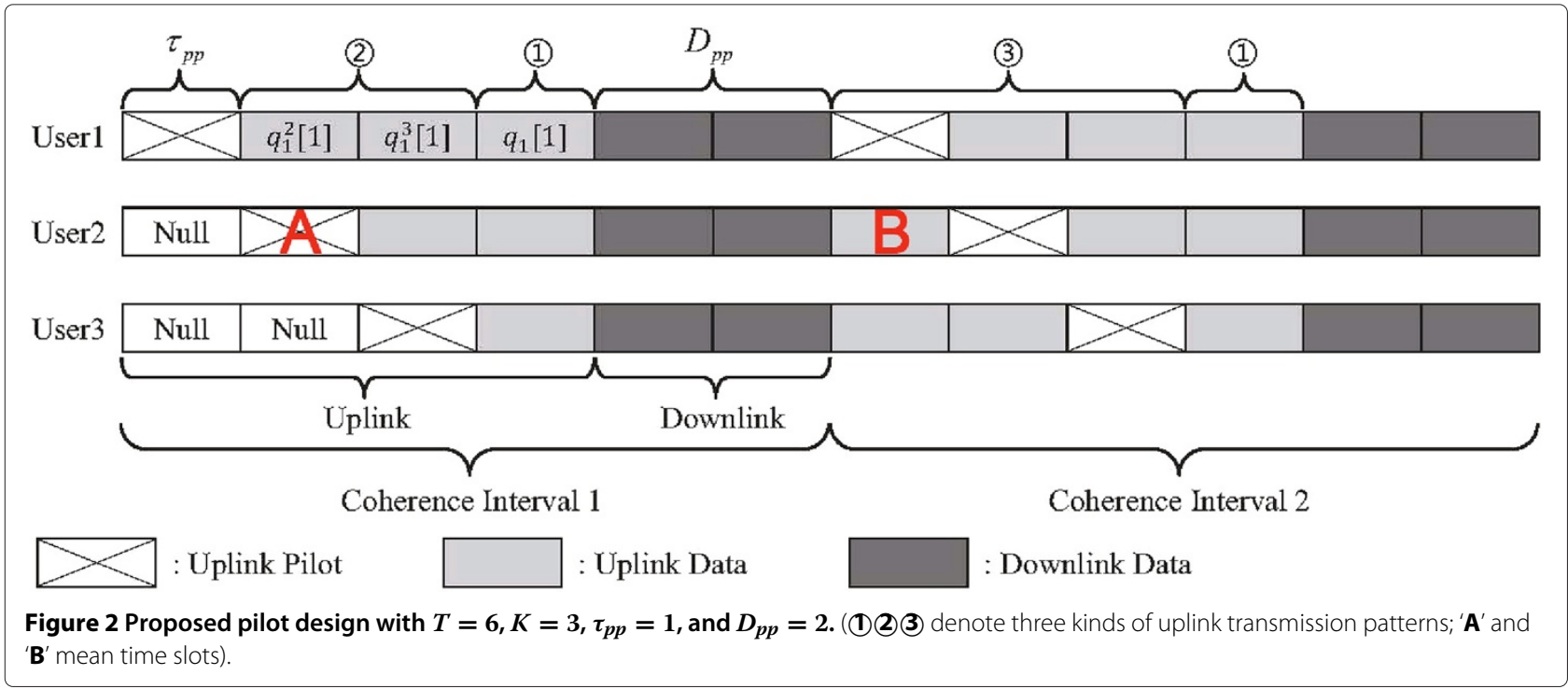


Table 1 Notational definition

\begin{tabular}{lcl}
\hline Symbols & Constraints & Meanings \\
\hline $\mathbf{y}^{k}[i]$ & $\mathbf{y}^{k}[i] \in \mathbb{C}^{M \times 1}$ & $\begin{array}{l}\text { Received signal at the BS when the } \\
k \text { th user transmits pilot }\end{array}$ \\
\hline $\mathbf{w}^{k}[i]$ & $\mathbf{w}^{k}[i] \in \mathbb{C}^{M \times 1}$ & $\begin{array}{l}\text { Unit variance AWGN when the } \\
k \text { th user transmits pilot }\end{array}$ \\
\hline$\varphi_{k}$ & $\varphi_{k} \in \mathbb{C}, \varphi_{k}^{*} \varphi_{k}=1$ & The $k$ th user's pilot \\
\hline$q_{t}^{k}[i]$ & $q_{t}^{k}[i] \in \mathbb{C}$ & The tth user's uplink data signal when \\
\hline$q_{t}[i]$ & $q_{t}[i] \in \mathbb{C}$ & the kth user transmits pilot \\
\hline$s_{t}[i]$ & $s_{t}[i] \in \mathbb{C}$ & The tth user's uplink data signal \\
\end{tabular}

AWGN, additive white Gaussian noise.

Once the BS gets the first user's channel estimate, the first user starts uplink data transmission. By exploiting the merit of massive MIMO, simultaneous pilot and data transmission of other users has little impact on the first user's uplink data detection.

Concerning the first user's uplink data detection when the $k \operatorname{th}(k>1)$ user transmits pilot, the received signal at the $\mathrm{BS}$ is

$$
\mathbf{y}^{k}[1]=\underbrace{\sqrt{\rho_{p}} \mathbf{g}_{k}[1] \varphi_{k}}_{\text {pilot }}+\underbrace{\sqrt{\rho_{u}} \sum_{t=1}^{k-1} \mathbf{g}_{t}[1] q_{t}^{k}[1]}_{\text {data interference }}+\mathbf{w}^{k}[1] .
$$

The BS applies maximum-ratio combining (MRC) since it is viable and common in massive MIMO uplink data reception $[2,16]$. The uplink data detection of the first user when the $k$ th user transmits pilot equals

$$
\begin{aligned}
\hat{q}_{1}^{k}[1]= & \frac{\hat{\mathbf{g}}_{1}^{H}[1] \mathbf{y}^{k}[1]}{\sqrt{\rho_{u}}\left\|\hat{\mathbf{g}}_{1}[1]\right\|^{2}} \\
\stackrel{(\mathrm{a})}{=} & \frac{\hat{\mathbf{g}}_{1}^{H}[1]\left(\hat{\mathbf{g}}_{1}[1]+\tilde{\mathbf{g}}_{1}[1]\right)}{\left\|\hat{\mathbf{g}}_{1}[1]\right\|^{2}} q_{1}^{k}[1]+\frac{\sqrt{\rho_{p}} \hat{\mathbf{g}}_{1}^{H}[1] \mathbf{g}_{k}[1]}{\sqrt{\rho_{u}}\left\|\hat{\mathbf{g}}_{1}[1]\right\|^{2}} \varphi_{k} \\
& +\sum_{t=2}^{k-1} \frac{\hat{\mathbf{g}}_{1}^{H}[1] \mathbf{g}_{t}[1]}{\left\|\hat{\mathbf{g}}_{1}[1]\right\|^{2}} q_{t}^{k}[1]+\frac{\hat{\mathbf{g}}_{1}^{H}[1] \mathbf{w}^{k}[1]}{\sqrt{\rho_{u}}\left\|\hat{\mathbf{g}}_{1}[1]\right\|^{2}} \\
& \stackrel{\text { a.s. }}{=} q_{1}^{k}[1],
\end{aligned}
$$

where we divide both the denominators and numerators in $\stackrel{\text { (a) }}{=}$ by $M$ and apply Lemma 1 , because $\hat{\mathbf{g}}_{1}[1]$ is independent of $\mathbf{g}_{t}[1](t \neq 1), \tilde{\mathbf{g}}_{1}[1]$, and $\mathbf{w}^{k}[1]$ from (1) and (2) according to the nature of MMSE estimation. Note that in a massive MIMO system, we assume that $M$ is large enough to meet Lemma 1.
Lemma 1. [16] Let $\mathbf{p}$ and $\mathbf{q}$ are two mutually independent $L \times 1$ vectors whose elements are i.i.d $\mathcal{C N}(0,1)$ random variables. Then $\lim _{L \rightarrow \infty} \mathbf{p}^{H} \mathbf{p} / L \stackrel{\text { a.s. }}{=} 1$ and $\lim _{L \rightarrow \infty} \mathbf{p}^{H} \mathbf{q} / L \stackrel{\text { a.s. }}{=} 0$, where $\stackrel{\text { a.s., }}{=}$ denotes almost sure convergence.

Then we consider the uplink data detection of the first user after the BS has obtained all users' channel estimates. The received signal at the BS when all users transmit uplink data is

$$
\mathbf{y}^{u}[1]=\sqrt{\rho_{u}} \sum_{j=1}^{K} \mathbf{g}_{j}[1] q_{j}[1]+\mathbf{z}[1]
$$

where $\mathbf{z}[1] \in \mathbb{C}^{M \times 1}$ is the additive white Gaussian noise. Following the steps in (4), the BS detects $\hat{q}_{1}[1]$ as follows:

$$
\hat{q}_{1}[1]=\frac{\hat{\mathbf{g}}_{1}^{H}[1] \mathbf{y}^{u}[1]}{\sqrt{\rho_{u}}\left\|\hat{\mathbf{g}}_{1}[1]\right\|^{2}} \stackrel{\text { a.s. }}{=} q_{1}[1] .
$$

Equations (4) and (6) show that the uplink data for the first user in the first coherence interval can be exactly detected in a massive MIMO system with the proposed pilot design.

\subsubsection{Uplink of the $k t h(k>1)$ user}

As for the other users in the first coherence interval, the main difference lies in the channel estimation period.

We first consider the $k$ th user's channel estimate. Given $\mathbf{y}^{k}[1]$ in (3), the BS removes the data interference caused by the $t$ th user from $\mathbf{y}^{k}[1]$ by using $\hat{\mathbf{g}}_{t}[1]$ and $\hat{q}_{t}^{k}[1]$ (as revealed in (4), $\hat{q}_{t}^{k}[1]$ is assumed to be accurate and equal 
to $q_{t}^{k}[1], k>t \geq 1$ ), which are obtained before the present period. The processed received signal is

$$
\begin{aligned}
\mathbf{r}^{k}[1] & =\left(\mathbf{y}^{k}[1]-\sqrt{\rho_{u}} \sum_{t=1}^{k-1} \hat{\mathbf{g}}_{t}[1] \hat{q}_{t}^{k}[1]\right) \varphi_{k}^{*} \\
& =\sqrt{\rho_{p}} \mathbf{g}_{k}[1]+\left(\sqrt{\rho_{u}} \sum_{t=1}^{k-1} \tilde{\mathbf{g}}_{t}[1] q_{t}^{k}[1]+\mathbf{w}^{k}[1]\right) \varphi_{k}^{*} \\
& =\sqrt{\rho_{p}} \mathbf{g}_{k}[1]+\mathbf{n}^{k}[1],
\end{aligned}
$$

where $\mathbf{n}^{k}[1]=\left(\sqrt{\rho_{u}} \sum_{t=1}^{k-1} \tilde{\mathbf{g}}_{t}[1] q_{t}^{k}[1]+\mathbf{w}^{k}[1]\right) \varphi_{k}^{*}$ means the residual interference and noise during the $k$ th user's channel estimation in the first coherence interval. Based on the assumption that $\left\{q_{t}^{k}[1]\right\}$ is an independent Gaussian sequence, assuming $\tilde{\mathbf{g}}_{t}[1] \sim \mathcal{C N}\left(\mathbf{0}, \varepsilon_{t, 1}^{2} \mathbf{I}_{M}\right)$, we have

$$
\mathrm{E}\left[\mathbf{n}^{k}[1]\left(\mathbf{n}^{k}[1]\right)^{H}\right]=\left(\rho_{u} \sum_{t=1}^{k-1} \varepsilon_{t, 1}^{2}+1\right) \mathbf{I} \triangleq \sigma_{\mathbf{n}^{k}[1]}^{2} \mathbf{I} .
$$

Obviously, $\mathbf{n}^{k}[1]$ is independent of $\mathbf{g}_{l}[1]$ for any $l(1 \leq$ $l \leq K)$, given $\mathbf{r}^{k}[1]$ in (7), the MMSE estimate of $\mathbf{g}_{k}[1]$ is

$$
\hat{\mathbf{g}}_{k}[1]=\mathbf{A}_{k}[1]\left(\mathbf{Q}_{k}[1]\right)^{-1} \mathbf{r}^{k}[1]
$$

where $\mathbf{A}_{k}[1]$ is defined as

$$
\begin{aligned}
\mathbf{A}_{k}[1] & =\mathrm{E}\left[\mathbf{g}_{k}[1]\left(\mathbf{r}^{k}[1]\right)^{H}\right] \\
& \stackrel{(b)}{=} \mathrm{E}\left[\mathbf{g}_{k}[1]\left(\sqrt{\rho_{p}} \mathbf{g}_{k}[1]+\mathbf{n}^{k}[1]\right)^{H}\right] \\
& =\sqrt{\rho_{p}} \beta_{k} \mathbf{I} .
\end{aligned}
$$

We exploit the independency between $\mathbf{g}_{k}[1]$ and $\mathbf{n}^{k}[1]$ in $\stackrel{(\mathrm{b})}{=}$ and also apply it in $\stackrel{(\mathrm{c})}{=}$ to obtain $\mathbf{Q}_{k}[1]$.

$$
\begin{aligned}
\mathbf{Q}_{k}[1] & =\mathrm{E}\left[\mathbf{r}^{k}[1]\left(\mathbf{r}^{k}[1]\right)^{H}\right] \\
& \stackrel{(\mathrm{c})}{=} \mathrm{E}\left[\left(\sqrt{\rho_{p}} \mathbf{g}_{k}[1]+\mathbf{n}^{k}[1]\right)\left(\sqrt{\rho_{p}} \mathbf{g}_{k}[1]+\mathbf{n}^{k}[1]\right)^{H}\right] \\
& =\left(\rho_{p} \beta_{k}+\sigma_{\mathbf{n}^{k}[1]}^{2}\right) \mathbf{I} .
\end{aligned}
$$

By substituting (10) and (11) into (9), we obtain

$$
\hat{\mathbf{g}}_{k}[1]=\frac{\sqrt{\rho_{p}} \beta_{k}}{\sigma_{\mathbf{n}^{k}[1]}^{2}+\rho_{p} \beta_{k}} \mathbf{r}^{k}[1] .
$$

Again, the channel can be decomposed as $\mathbf{g}_{k}[1]=$ $\tilde{\mathbf{g}}_{k}[1]+\hat{\mathbf{g}}_{k}[1]$. From the properties of MMSE estimation, $\hat{\mathbf{g}}_{k}[1] \sim \mathcal{C N}\left(\mathbf{0}, \sigma_{k, 1}^{2} \mathbf{I}_{M}\right)$ and $\tilde{\mathbf{g}}_{k}[1] \sim \mathcal{C N}\left(\mathbf{0}, \varepsilon_{k, 1}^{2} \mathbf{I}_{M}\right)$ is the independent estimation error, where $\varepsilon_{k, 1}^{2}=\beta_{k}-\sigma_{k, 1}^{2}$ and

$$
\sigma_{k, 1}^{2}=\frac{\rho_{p} \beta_{k}^{2}}{\sigma_{\mathbf{n}^{k}[1]}^{2}+\rho_{p} \beta_{k}} .
$$

Then we concern the uplink data detection of the $k$ th user when the $k_{1} \operatorname{th}\left(k_{1}>k\right)$ user transmits pilot. From (7) and (12), $\hat{\mathbf{g}}_{k}[1]$ and $\mathbf{g}_{t}[1](t \neq k)$ are independent due to the fact that $\mathbf{g}_{t}[1]$ is independent of $\mathbf{g}_{k}[1]$ and $\mathbf{n}^{k}[1]$. Following the steps in (4), the uplink data detection for $k_{1}>k$ equals

$$
\hat{q}_{k}^{k_{1}}[1]=\frac{\hat{\mathbf{g}}_{k}^{H}[1] \mathbf{y}^{k_{1}}[1]}{\sqrt{\rho_{u}}\left\|\hat{\mathbf{g}}_{k}[1]\right\|^{2}} \stackrel{\text { a.s. }}{=} q_{k}^{k_{1}}[1] .
$$

As for the uplink data detection of the $k^{\text {th }}$ user when all users transmit uplink data, we can achieve a similar result to (6):

$$
\hat{q}_{k}[1]=\frac{\hat{\mathbf{g}}_{k}^{H}[1] \mathbf{y}^{u}[1]}{\sqrt{\rho_{u}\left\|\hat{\mathbf{g}}_{k}[1]\right\|^{2}}} \text { a.s. } q_{k}[1] .
$$

From (14) and (15), the uplink data for the $k$ th user in the first coherence interval can also be precisely detected in a massive MIMO system with the proposed pilot design.

\subsubsection{Downlink of all users}

After all users complete the uplink pilot and data transmission, the BS starts downlink data transmission, which is the same for all users. The BS applies matched filter (MF)-based beamforming since it works well enough in massive MIMO downlink data transmission [17]. Without loss of generality, we consider the downlink data transmission of the $k$ th user. The $k$ th user receives

$$
y_{k}^{d}[1]=\sqrt{\rho_{d}} \sum_{j=1}^{K} \mathbf{g}_{k}^{H}[1] \mathbf{p}_{j}[1] s_{j}[1]+v_{k}[1],
$$

where $y_{k}^{d}[1] \in \mathbb{C}$ is the receiving signal and $v_{k}[1] \in \mathbb{C}$ is the unit AWGN. $\mathbf{p}_{j}[1]$ is the beamforming vector for the $j$ th user and is defined as

$$
\mathbf{p}_{j}[1]=\frac{\hat{\mathbf{g}}_{j}[1]}{\sqrt{K}\left\|\hat{\mathbf{g}}_{j}[1]\right\|} .
$$

Because $\hat{\mathbf{g}}_{k}[1]$ is independent of $\tilde{\mathbf{g}}_{k}[1]$ and $\mathbf{g}_{k}[1]$ is independent of $\hat{\mathbf{g}}_{j}[1](j \neq k)$, we apply it to equality $\stackrel{(\mathrm{d})}{=}$ in the following and can obtain the downlink data estimate of the $k$ th user: 


$$
\begin{aligned}
\hat{s}_{k}[1]= & \frac{\sqrt{K} y_{k}^{d}[1]}{\sqrt{\rho_{d}}\left\|\hat{\mathbf{g}}_{k}[1]\right\|} \\
\stackrel{(\mathrm{d})}{=} & \frac{\left(\hat{\mathbf{g}}_{k}[1]+\tilde{\mathbf{g}}_{k}[1]\right)^{H} \hat{\mathbf{g}}_{k}[1]}{\left\|\hat{\mathbf{g}}_{k}[1]\right\|^{2}} s_{k}[1] \\
& +\sum_{j=1, j \neq k}^{K} \frac{\mathbf{g}_{k}^{H}[1]}{\left\|\hat{\mathbf{g}}_{k}[1]\right\|} \frac{\hat{\mathbf{g}}_{j}[1]}{\left\|\hat{\mathbf{g}}_{j}[1]\right\|} s_{j}[1]+\frac{\sqrt{K} v_{k}[1]}{\sqrt{\rho_{d}\left\|\hat{\mathbf{g}}_{k}[1]\right\|}} \\
& \stackrel{\text { a.s. }}{=} s_{k}[1],
\end{aligned}
$$

where we divide both the denominator and numerator of the last term in $\stackrel{(\mathrm{d})}{=}$ by $\sqrt{M}$ and it vanishes as $M \rightarrow \infty$. Equation (18) shows that the downlink data can also be accurately detected.

\subsection{Communication in the $i$ th $(i>1)$ coherence interval}

Note that, different from the first coherence interval, all users show the same transmission pattern in the $i$ th $(i>1)$ coherence interval as shown in Figure 2. Hence, without loss of generality, we take the $k$ th user for instance. Its channel estimation is contaminated by all the other users' uplink data. As for the uplink and downlink data transmissions, they are similar to the procedures elaborated for the first coherence interval in the above subsection and we omit the detailed description due to space limitation. To be concise, we here briefly introduce the processing procedure of channel estimation in the following.

The received signal at the BS when the $k$ th user transmits a pilot is

$$
\mathbf{y}^{k}[i]=\sqrt{\rho_{p}} \mathbf{g}_{k}[i] \varphi_{k}+\sqrt{\rho_{u}} \sum_{t=1, t \neq k}^{K} \mathbf{g}_{t}[i] q_{t}^{k}[i]+\mathbf{w}^{k}[i] .
$$

Then the BS gets the uplink data estimate $q_{t}^{k}[i]$ of the $t \operatorname{th}(t \neq k)$ user:

$$
\hat{q}_{t}^{k}[i]=\left\{\begin{array}{cl}
\frac{\hat{\mathbf{g}}_{t}^{H}[i] \mathbf{y}^{k}[i]}{\sqrt{\rho_{u}}\left\|\hat{\mathbf{g}}_{t}[i]\right\|^{2}} & , 1 \leq t \leq k-1 \\
\frac{\hat{\mathbf{g}}_{t}^{H}[i-1] \mathbf{y}^{k}[i]}{\sqrt{\rho_{u}}\left\|\hat{\mathbf{g}}_{t}[i-1]\right\|^{2}} & , k \leq t \leq K,
\end{array}\right.
$$

which uses the known channel estimate from the present coherence interval when $1 \leq t \leq k-1$ and uses that from the previous one when $k \leq t \leq K$. Hence (20) is separately expressed based on two conditions.

Here, we assume that the variation of channel vectors during a coherence interval is ignorable. Any two channel vectors between the same user and BS can be treated as approximately equal if they locate within a distance of $T$. Take the channel vector from the second user to BS for example. Let $\mathbf{g}_{A}$ and $\mathbf{g}_{B}$ respectively denote the channel vectors at time slots ' $A$ ' and 'B' in Figure 2. Since the distance between 'A' and 'B' is no larger than $T$, it is reasonable to approximately treat $\mathbf{g}_{B}$ as $\mathbf{g}_{A}$, i.e., $\mathbf{g}_{B} \approx \mathbf{g}_{A}$. Therefore, as shown in (20) for the $k \leq t \leq K$ case, the BS utilizes the estimate of $\mathbf{g}_{A}$ to detect the second user's uplink data at time slot ' $\mathrm{B}$.' This is different from the uplink data detection in (14), which is expressed based on only one condition. As for the following channel estimation process, it is performed in the similar way to that in Section 3.1. In this way, the $k \leq t \leq K$ case for (20) establishes the main difference of the analysis for the $i$ th $(i>1)$ coherence interval from that of Section 3.1.

Equation (20) can also be extended similarly to the form in (4) and then we arrive at the conclusion that $\hat{q_{t}^{k}}[i] \stackrel{\text { a.s. }}{=} q_{t}^{k}[i]$.

Similar to the procedure in (7), the BS first removes the data contamination from the received signal and generates

$$
\begin{aligned}
\mathbf{r}^{k}[i]= & {\left[\mathbf{y}^{k}[i]-\sqrt{\rho_{u}}\left(\sum_{t=1}^{k-1} \hat{\mathbf{g}}_{t}[i]+\sum_{t=k+1}^{K} \hat{\mathbf{g}}_{t}[i-1]\right) \hat{q}_{t}^{k}[i]\right] \varphi_{k}^{*} } \\
= & \sqrt{\rho_{p}} \mathbf{g}_{k}[i]+\left(\sqrt{\rho_{u}} \sum_{t=1}^{k-1} \tilde{\mathbf{g}}_{t}[i] \hat{q}_{t}^{k}[i]\right. \\
& \left.+\sqrt{\rho_{u}} \sum_{t=k+1}^{K} \tilde{\mathbf{g}}_{t}[i-1] \hat{q}_{t}^{k}[i]+\mathbf{w}^{k}[i]\right) \varphi_{k}^{*} \\
= & \sqrt{\rho_{p}} \mathbf{g}_{k}[i]+\mathbf{n}^{k}[i] .
\end{aligned}
$$

where $\mathbf{n}^{k}[i]$ means the residual interference and noise during the $k$ th user's channel estimation in the $i$ th $(i>1)$ coherence interval. Following the steps in (8), (9), (10), and (11), the BS computes

$$
\begin{aligned}
\mathrm{E}\left[\mathbf{n}^{k}[i]\left(\mathbf{n}^{k}[i]\right)^{H}\right] & =\left(\rho_{u} \sum_{t=1}^{k-1} \varepsilon_{t, i}^{2}+\rho_{u} \sum_{t=k+1}^{K} \varepsilon_{t, i-1}^{2}+1\right) \mathbf{I} \\
& \triangleq \sigma_{\mathbf{n}^{k}[i]}^{2} \mathbf{I}
\end{aligned}
$$

$$
\mathbf{A}_{k}[i]=\mathrm{E}\left[\mathbf{g}_{k}[i]\left(\mathbf{r}^{k}[i]\right)^{H}\right]=\sqrt{\rho_{p}} \beta_{k} \mathbf{I},
$$

and

$$
\begin{aligned}
\mathbf{Q}_{k}[i] & =\mathrm{E}\left[\mathbf{r}^{k}[i]\left(\mathbf{r}^{k}[i]\right)^{H}\right] \\
& =\mathrm{E}\left[\left(\sqrt{\rho_{p}} \mathbf{g}_{k}[i]+\mathbf{n}^{k}[i]\right)\left(\sqrt{\rho_{p}} \mathbf{g}_{k}[i]+\mathbf{n}^{k}[i]\right)^{H}\right] \\
& =\left(\rho_{p} \beta_{k}+\sigma_{\mathbf{n}^{k}[i]}^{2}\right) \mathbf{I},
\end{aligned}
$$


Then the BS gets an MMSE estimate:

$$
\hat{\mathbf{g}}_{k}[i]=\frac{\sqrt{\rho_{p}} \beta_{k}}{\sigma_{\mathbf{n}^{k}[i]}^{2}+\rho_{p} \beta_{k}} \mathbf{r}^{k}[i] .
$$

Again, the channel can be decomposed as $\mathbf{g}_{k}[i]=$ $\tilde{\mathbf{g}}_{k}[i]+\hat{\mathbf{g}}_{k}[i]$. From the properties of MMSE estimation, $\hat{\mathbf{g}}_{k}[i] \sim \mathcal{C N}\left(\mathbf{0}, \sigma_{k, i}^{2} \mathbf{I}_{M}\right), \tilde{\mathbf{g}}_{k}[i] \sim \mathcal{C N}\left(\mathbf{0}, \varepsilon_{k, i}^{2} \mathbf{I}_{M}\right)$ is the independent estimation error, where $\varepsilon_{k, i}^{2}=\beta_{k}-\sigma_{k, i}^{2}$ and

$$
\sigma_{k, i}^{2}=\frac{\rho_{p} \beta_{k}^{2}}{\sigma_{\mathbf{n}^{k}[i]}^{2}+\rho_{p} \beta_{k}} .
$$

Equation (26) is the unified form of $\sigma_{k, i}^{2}$ for any $i$ th $(i \geq 1)$ coherence interval by comparing (13) with (26). Analysis in the next section shows that $\sigma_{k, i}^{2}$ will be useful in the performance analysis of our proposed pilot design.

\section{Performance analysis}

Given the processing procedure elaborated in the above section, we are now ready to conduct theoretical performance analysis of our proposed scheme. From Figure 2, the proposed pilot design saves more resource for data transmission compared with the conventional orthogonal one when $K$ grows large along with $M$. However, due to the semi-orthogonal pilot pattern, SIC-based channel estimation is adopted, leading to a larger estimation error than the conventional one. In this section, performance analysis is presented to show explicitly the potential benefits that can be achieved by our proposed pilot design.

In this section, we analyze the performance of the massive MIMO systems in terms of both the uplink and downlink achievable rates. The approximation of the $k$ th user's achievable rate [18] is defined as

$$
R_{k}=\log _{2}\left(1+\gamma_{k}\right)=\log _{2}\left(1+\frac{S}{I+N}\right)
$$

where $\gamma_{k}$ is the associated signal-to-interference-noiseratio (SINR); $S, I$, and $N$ stand for the power of signal, interference, and noise, respectively. Next the achievable rate is calculated by evaluating the power of these items term by term.

\subsection{Downlink analysis}

Here we first conduct downlink performance analysis since it is much simpler than the uplink one. Assume that the $k$ th user is of interest. Review the process of downlink communication in (16) and replace $\mathbf{p}_{j}[1]$ with $\mathbf{p}_{j}[i]$ $(i \geq 1)$ to formulate the downlink communication for any $i$ th coherence intervals:

$$
\begin{aligned}
y_{k}^{d}[i]= & \underbrace{\sqrt{\rho_{d}} \mathbf{g}_{k}^{H}[i] \mathbf{p}_{k}[i] s_{k}[i]}_{\text {signal }(S)} \\
& +\underbrace{\sqrt{\rho_{d}} \sum_{j=1, j \neq k}^{K} \mathbf{g}_{k}^{H}[i] \mathbf{p}_{j}[i] s_{j}[i]}_{\text {interference }(I)}+\underbrace{v_{k}[i]}_{\text {noise }(N)},
\end{aligned}
$$

where the signal, interference, and noise terms are marked with $S, I$, and $N$, and $\mathbf{g}_{k}^{H}[i] \mathbf{p}_{k}[i]$ is assumed to be accurately estimated at the $k$ th user.

First, we derive the power of the intended signal in (28), denoted by $S_{k, i}^{d}$. By applying Khintchine's law of large numbers [19] and with some basic manipulations, we have the following equality in $\stackrel{(\mathrm{e})}{=}$ with high probability for large $M$. Thus we arrive at

$$
\begin{aligned}
S_{k, i}^{d} & =\rho_{d}\left|\mathbf{g}_{k}^{H}[i] \mathbf{p}_{k}[i]\right|^{2} \\
& =\frac{\rho_{d}}{\alpha_{k, i}^{2} M K}\left(\hat{\mathbf{g}}_{k}[i]+\tilde{\mathbf{g}}_{k}[i]\right)^{H} \hat{\mathbf{g}}_{k}[i] \hat{\mathbf{g}}_{k}^{H}[i]\left(\hat{\mathbf{g}}_{k}[i]+\tilde{\mathbf{g}}_{k}[i]\right) \\
& \stackrel{(\mathrm{e})}{=} \frac{\rho_{d}}{\alpha_{k, i}^{2} M K} \mathrm{E}\left[\left|\hat{\mathbf{g}}_{k}^{H}[i] \hat{\mathbf{g}}_{k}[i]\right|^{2}+\left|\tilde{\mathbf{g}}_{k}^{H}[i] \hat{\mathbf{g}}_{k}[i]\right|^{2}\right] \\
& \stackrel{(\mathrm{f})}{=} \frac{\rho_{d}}{\alpha_{k, i}^{2} M K}\left[\left(M^{2}+M\right) \sigma_{k, i}^{4}+M \varepsilon_{k, i}^{2} \sigma_{k, i}^{2}\right] \\
& =\frac{\rho_{d}(M+1) \sigma_{k, i}^{2}+\rho_{d} \varepsilon_{k, i}^{2}}{K} \\
& \stackrel{(\mathrm{h})}{=} \frac{\rho_{d} M \sigma_{k, i}^{2}+\rho_{d} \beta_{k}}{K},
\end{aligned}
$$

where $\mathrm{E}[\cdot]$ means the expectation operator and $\mathbf{p}_{k}[i]$ is the beamforming vector:

$$
\mathbf{p}_{k}[i]=\frac{\hat{\mathbf{g}}_{k}[i]}{\sqrt{K}\left\|\hat{\mathbf{g}}_{k}[i]\right\|}=\frac{\hat{\mathbf{g}}_{k}[i]}{\alpha_{k, i} \sqrt{M K}} .
$$

The scalar $\alpha_{k . i}=\frac{\left\|\hat{\mathbf{s}}_{k}[i]\right\|}{\sqrt{M}}$ is a normalization factor [10]. By using Lemma 1 , we can get the value of $\alpha_{k, i}$ as $M \rightarrow \infty$ :

$$
\lim _{M \rightarrow \infty} \alpha_{k, i}^{2}=\lim _{M \rightarrow \infty} \hat{\mathbf{g}}_{k}^{H}[i] \hat{\mathbf{g}}_{k}[i] \stackrel{\text { a.s. }}{=} \sigma_{k, i}^{2} .
$$

Besides, $\stackrel{(\mathrm{f})}{=}$ exploits Lemma 2 in the following and $\stackrel{(\mathrm{h})}{=}$ utilizes the fact that $\sigma_{k, i}^{2}+\varepsilon_{k, i}^{2}=\beta_{k}$.

Lemma 2. Let $\mathbf{x}$ and $\mathbf{y}$ are two mutually independent $L \times 1$ vectors whose elements are i.i.d $\mathbb{C N}\left(0, \sigma^{2}\right)$ random variables. Then $\mathrm{E}\left[\left|\mathbf{x}^{H} \mathbf{x}\right|^{2}\right]=\left(L^{2}+L\right) \sigma^{4}$ and $\mathrm{E}\left[\left|\mathbf{x}^{H} \mathbf{y}\right|^{2}\right]=$ $L \sigma^{4}$.

Proof. : It can be directly obtained by correlating the vectors in the element-wise way. 
Using a similar technique to (29), we have the power of the interference in (28), denoted by $I_{k, i}^{d}$, as follows:

$$
\begin{aligned}
I_{k, i}^{d} & =\sum_{j=1, j \neq k}^{K} \rho_{d}\left|\mathbf{g}_{k}^{H}[i] \mathbf{p}_{j}[i]\right|^{2} \\
& =\sum_{j=1, j \neq k}^{K} \frac{\rho_{d}}{\alpha_{j, i}^{2} M K} \mathrm{E}\left[\left|\mathbf{g}_{k}^{H}[i] \hat{\mathbf{g}}_{j}[i]\right|^{2}\right] \\
& \stackrel{(\mathrm{i})}{=} \sum_{j=1, j \neq k}^{K} \frac{\rho_{d}}{\alpha_{j, i}^{2} M K} M \beta_{k} \sigma_{j, i}^{2} \\
& =\frac{(K-1) \rho_{d} \beta_{k}}{K},
\end{aligned}
$$

where $\stackrel{(\mathbf{i})}{=}$ is based on the independency between $\mathbf{g}_{k}[i]$ and $\hat{\mathbf{g}}_{j}[i](k \neq j)$ and the fact that the variance of $\mathbf{g}_{j}[i]$ is $\beta_{j} \mathbf{I}_{M}$.

The noise power in (28) can be readily obtained by $N_{k, i}^{d}=\mathrm{E}\left[v_{k}^{*}[i] v_{k}[i]\right]=1$. Then the downlink achievable rate of the $k$ th user in the $i$ th coherence interval is

$$
R_{k, i}^{d}=\log _{2}\left(1+\frac{\rho_{d} M \sigma_{k, i}^{2}+\rho_{d} \beta_{k}}{(K-1) \rho_{d} \beta_{k}+K}\right) .
$$

\subsection{Uplink analysis}

There are three kinds of uplink transmission patterns in massive MIMO systems with the proposed pilot design. The first kind of uplink communication contains only uplink data, i.e., case (1) in Figure 2, while the other two kinds of uplink communications, i.e., cases (2) and (3) in Figure 2, cover both the uplink data and pilot. Here we take the first kind of uplink communication as an example since the respective analysis of the other two kinds of uplink communications are similar.

Assume that the $k$ th user is of interest. Write down the MRC processed received signal at the BS based on (5) and replace ' 1 ' with ' $i$ ' $(i \geq 1)$ to formulate the uplink data transmission for any $i$ th coherence interval. It gives

$$
\begin{aligned}
y_{k}^{u}[i]= & \underbrace{\sqrt{\rho_{u}} \hat{\mathbf{g}}_{k}^{H}[i] \hat{\mathbf{g}}_{k}[i] q_{k}[i]}_{\text {signal }(S)}+\underbrace{\sqrt{\rho_{u}} \hat{\mathbf{g}}_{k}^{H}[i] \tilde{\mathbf{g}}_{k}[i] q_{k}[i]}_{\text {interference }\left(I_{1}\right)} \\
& +\underbrace{\sqrt{\rho_{u}} \sum_{j=1, j \neq k}^{K} \hat{\mathbf{g}}_{k}^{H}[i] \mathbf{g}_{j}[i] q_{j}[i]}_{\text {interference }\left(I_{2}\right)}+\underbrace{\hat{\mathbf{g}}_{k}^{H}[i] \mathbf{z}[i]}_{\text {noise }(N)}
\end{aligned}
$$

where the signal, interference (composed of two parts) and noise terms are marked with $S, I_{1}, I_{2}$, and $N$. By applying the similar analytical procedure for the downlink, it is not hard to calculate the power of the desired signal, the interference and the noise in (34) term by term, which are respectively denoted by $S_{k, i}^{u}, I_{k, i}^{u}$, and $N_{k, i}^{u}$. The power of the desired signal is

$$
\begin{aligned}
S_{k, i}^{u} & =\rho_{u}\left|\hat{\mathbf{g}}_{k}^{H}[i] \hat{\mathbf{g}}_{k}[i]\right|^{2} \\
& =\rho_{u}\left(M^{2}+M\right) \sigma_{k, i}^{4}
\end{aligned}
$$

And the power of the interference in (34) is

$$
\begin{aligned}
I_{k, i}^{u} & =\rho_{u}\left|\hat{\mathbf{g}}_{k}^{H}[i] \tilde{\mathbf{g}}_{k}[i]\right|^{2}+\rho_{u} \sum_{j=1, j \neq k}^{K}\left|\hat{\mathbf{g}}_{k}^{H}[i] \mathbf{g}_{j}[i]\right|^{2} \\
& =\rho_{u} M \sigma_{k, i}^{2} \varepsilon_{k, i}^{2}+\rho_{u} \sum_{j=1, j \neq k}^{K} M \sigma_{k, i}^{2} \beta_{j} \\
& =\rho_{u} M \sigma_{k, i}^{2} \sum_{j=1}^{K} \beta_{j}-\rho_{u} M \sigma_{k, i}^{4} .
\end{aligned}
$$

Then the noise power in (34) is readily achieved:

$$
N_{k, i}^{u}=\left|\hat{\mathbf{g}}_{k}^{H}[i] \hat{\mathbf{g}}_{k}[i]\right|=M \sigma_{k, i}^{2}
$$

Substituting the analyzed $S_{k, i}^{u}, I_{k, i}^{u}$, and $N_{k, i}^{u}$ into (27), the $k$ th user's uplink achievable rate of case (1) in Figure 2 is

$$
R_{k, i}^{u}=\log _{2}\left(1+\frac{\rho_{u}(M+1) \sigma_{k, i}^{2}}{\rho_{u} \sum_{j=1}^{K} \beta_{j}-\rho_{u} \sigma_{k, i}^{2}+1}\right) \text {. }
$$

As for the second kind of uplink communication, we can write down the processed receiving signal for the $k$ th user during the $k_{1}$ th user's first channel estimation as follows:

$$
\begin{aligned}
y_{k}^{u_{k_{1}}}[1]= & \underbrace{\sqrt{\rho_{u}} \hat{\mathbf{g}}_{k}^{H}[1] \hat{\mathbf{g}}_{k}[1] q_{k}^{k_{1}}[1]}_{\text {signal }(S)}+\underbrace{\sqrt{\rho_{u}} \hat{\mathbf{g}}_{k}^{H}[1] \tilde{\mathbf{g}}_{k}[1] q_{k}^{k_{1}}[1]}_{\text {interference }\left(I_{1}\right)} \\
& +\underbrace{\sqrt{\rho_{p}} \hat{\mathbf{g}}_{k}^{H}[1] \mathbf{g}_{k_{1}}[1] \varphi_{k_{1}}}_{\text {interference }\left(I_{2}\right)} \\
& +\underbrace{\sqrt{\rho_{u}} \sum_{t=1, t \neq k}^{k_{1}-1} \hat{\mathbf{g}}_{k}^{H}[1] \mathbf{g}_{t}[1] q_{t}^{k_{1}}[1]}_{\text {interference }\left(I_{3}\right)}+\underbrace{\hat{\mathbf{g}}_{k}^{H}[1] \mathbf{w}^{k_{1}}[1]}_{\text {noise }(N)} .
\end{aligned}
$$

The main difference of (39) from (34) lies in the interference term. Hence we only compute the power of the interference, denoted by $I_{k, 1}^{u_{k_{1}}}$ : 


$$
\begin{aligned}
I_{k, 1}^{u_{k_{1}}}= & \rho_{u}\left|\hat{\mathbf{g}}_{k}^{H}[1] \tilde{\mathbf{g}}_{k}[1]\right|^{2}+\rho_{u} \sum_{t=1, t \neq k}^{k_{1}-1}\left|\hat{\mathbf{g}}_{k}^{H}[1] \mathbf{g}_{t}[1]\right|^{2} \\
& +\rho_{p}\left|\hat{\mathbf{g}}_{k}^{H}[1] \mathbf{g}_{k_{1}}[1]\right|^{2} \\
= & \rho_{u} M \sigma_{k, 1}^{2} \varepsilon_{k, 1}^{2}+\rho_{u} \sum_{t=1, t \neq k}^{k_{1}-1} M \sigma_{k, 1}^{2} \beta_{t}+\rho_{p} M \sigma_{k, 1}^{2} \beta_{k_{1}} \\
= & M \sigma_{k, 1}^{2}\left(\rho_{u} \sum_{t=1}^{k_{1}-1} \beta_{t}-\rho_{u} \sigma_{k, 1}^{2}+\rho_{p} \beta_{k_{1}}\right) .
\end{aligned}
$$

Then the $k$ th user's uplink achievable rate when the $k_{1}$ th $\left(k_{1}>k\right)$ user transmits a pilot in the first coherence interval is

$$
R_{k, 1}^{u_{k_{1}}}=\log _{2}\left(1+\frac{\rho_{u}(M+1) \sigma_{k, 1}^{2}}{\rho_{u} \sum_{t=1}^{k_{1}-1} \beta_{t}-\rho_{u} \sigma_{k, 1}^{2}+\rho_{p} \beta_{k_{1}}+1}\right)
$$

Further concerning the third kind of uplink communication in a similar way, we can also get the uplink rate of the $k$ th user when the $k_{1}$ th user transmits pilot in the $i$ th $(i>1)$ coherence interval is

$$
R_{k, i}^{u_{k_{1}}}=\log _{2}\left(1+\frac{\rho_{u}(M+1) \sigma^{2}}{\rho_{u} \sum_{t=1, t \neq k_{1}}^{K} \beta_{t}-\rho_{u} \sigma^{2}+\rho_{p} \beta_{k_{1}}+1}\right),
$$

where $\sigma^{2}=\left\{\begin{array}{rr}\sigma_{k, i}^{2}, & k<k_{1} \\ \sigma_{k, i-1}^{2}, & k>k_{1}\end{array}\right.$.

\subsection{Performance evaluation}

Observing (33), (38), (41), and (42), the channel estimation accuracy $\sigma_{k, i}^{2}$ has an influential effect on the uplink and downlink achievable rates. Obviously, The channel estimates of the conventional one are more accurate than those of our proposed one. However, an inherent merit of the proposed one is that it provides more available resources for data communication, which is implied in (41) and (42). It makes a difference in performance evaluation. Assume that the number of concerned coherence intervals is $N_{c}$. Then from Figure 2, the uplink achievable rate of the proposed one, in terms of $\mathrm{bps} / \mathrm{Hz}$, is

$$
\begin{aligned}
\tilde{R}_{p p}^{u}= & \frac{1}{T N_{c}}(\underbrace{\sum_{k_{1}=2}^{K} \sum_{k_{2}=1}^{k_{1}-1} R_{k_{2}, 1}^{u_{k_{1}}}}_{\text {case (2) }}+\underbrace{\sum_{i=2}^{N_{c}} \sum_{k_{1}=1}^{K} \sum_{k_{2}=1, k_{2} \neq k_{1}}^{K} R_{k_{2}, i}^{u_{k_{1}}}}_{\text {case (3) }} \\
& +\underbrace{\left(T-K-D_{p p}\right) \sum_{i=1}^{N_{c}} \sum_{k=1}^{K} R_{k, i}^{u}}_{\text {case }(1)}) .
\end{aligned}
$$

Meanwhile, from (33), the downlink achievable rate (bps $/ \mathrm{Hz}$ ) of the proposed one is

$$
\tilde{R}_{p p}^{d}=\frac{1}{T N_{c}}\left(D_{p p} \sum_{i=1}^{N_{c}} \sum_{k=1}^{K} R_{k, i}^{d}\right) .
$$

For comparison, we also provide the uplink and downlink achievable rates $\tilde{R}_{o p}^{u}$ and $\tilde{R}_{o p}^{d}$ (bps/Hz) of the conventional orthogonal one in Figure 1 as follows:

$$
\begin{aligned}
& \tilde{R}_{o p}^{u}=\frac{1}{T N_{c}}\left(\left(T-K-D_{o p}\right) N_{c} \sum_{k=1}^{K} R_{o p, k}^{u}\right), \\
& \tilde{R}_{o p}^{d}=\frac{1}{T N_{c}}\left(D_{o p} N_{c} \sum_{k=1}^{K} R_{o p, k}^{d}\right),
\end{aligned}
$$

where the $k$ th user's uplink and downlink achievable rates of the conventional orthogonal pilots $R_{o p, k}^{u}$ and $R_{o p, k}^{d}$ are

$$
\begin{aligned}
& R_{o p, k}^{u}=\log _{2}\left(\frac{\rho_{u}(M+1) \sigma_{o p, k}^{2}}{\rho_{u} \sum_{j=1}^{K} \beta_{j}-\rho_{u} \sigma_{o p, k}^{2}+1}\right), \\
& R_{o p, k}^{d}=\log _{2}\left(\frac{\rho_{d} M \sigma_{o p, k}^{2}+\rho_{d} \beta_{k}}{(K-1) \rho_{d} \beta_{k}+K}\right) .
\end{aligned}
$$

Here, the variance of channel estimate $\sigma_{o p, k}^{2}$ for the conventional orthogonal pilots as shown in Figure 1 can be easily obtained and $\sigma_{o p, k}^{2}=\frac{\rho_{p} \tau_{o p} \beta_{k}^{2}}{1+\rho_{p} \tau_{o p} \beta_{k}}[16]$.

Remark 2. By comparing (43) with (45), the additional uplink data transmission, i.e., cases (2) and (3) in Figure 2, has an influential positive effect on the uplink achievable rate of the proposed pilot design for a small $T$. Though at a cost of channel estimation accuracy, however, by exploiting the merit of massive MIMO, the proposed pilot design can achieve a better tradeoff between pilot resource consumption and channel estimation accuracy. It outperforms the conventional one in many scenarios. 
Remark 3. Note that from the elaboration in Section 3, compared with the conventional orthogonal pilot design, the main difference of our proposed one lies in the subtraction of data interference from the received signal at the BS, which can be easily completed without much additional complexity.

For a better view of the system performance comparison, $C_{p p}^{s}=\tilde{R}_{p p}^{d}+\tilde{R}_{p p}^{u}$ and $C_{o p}^{s}=\tilde{R}_{o p}^{d}+\tilde{R}_{o p}^{u}$ are introduced to respectively stand for the system overall achievable rates of the proposed pilot design and the conventional one.

\section{Asymptotic performance analysis}

In this section, we will look at the performance at asymptotically low and high pilot and data SNRs. For simplicity, assume that $\frac{\rho_{u}}{\rho_{p}}$ and $\frac{\rho_{d}}{\rho_{p}}$ are fixed. Hence, $\rho_{u} \rightarrow 0$ and $\rho_{d} \rightarrow 0$ as $\rho_{p} \rightarrow 0$, and it is likewise as $\rho_{p} \rightarrow \infty$. We can use $\rho$ to stand for $\rho_{p}, \rho_{u}$, and $\rho_{d}$ when considering asymptotically low and high SNRs. Furthermore, the SNR of our proposed pilot design is defined as $\rho_{p p}=\frac{2 T N_{c}}{2 T N_{c}-K+1} \rho_{o p}$ to provide an equal overall system power, where $\rho_{o p}$ denotes the SNR of the conventional orthogonal one.

\subsection{High SNR analysis}

In order to evaluate the performance of two pilot designs at an asymptotically high SNR, we first reformulate the SINR value in (38) by some manipulations as follows:

$$
\begin{aligned}
\gamma_{k, i}^{u} & =\frac{\rho_{p p}(M+1) \sigma_{k, i}^{2}}{\rho_{p p} \sum_{j=1}^{K} \beta_{j}-\rho_{p p} \sigma_{k, i}^{2}+1} \\
& =-(M+1)+\frac{(M+1)\left(\rho_{p p} \sum_{j=1}^{K} \beta_{j}+1\right)}{\rho_{p p} \sum_{j=1}^{K} \beta_{j}+1-\rho_{p p} \sigma_{k, i}^{2}} \\
& =-(M+1)+\frac{(M+1)}{1-\lambda_{p p, k}},
\end{aligned}
$$

where $\lambda_{p p, k}=\frac{\beta_{k}^{2}}{\left(\frac{1}{\rho_{p p}}+\sum_{j=1}^{K} \beta_{j}\right)\left(\frac{\sigma^{n^{k}[i]}}{\rho_{p p}}+\beta_{k}\right)}$ is from (26). Following the steps in (49), the SINR value in (47) is

$$
\begin{aligned}
& \gamma_{o p, k}^{u}= \frac{\rho_{o p}(M+1) \sigma_{o p, k}^{2}}{\rho_{o p} \sum_{j=1}^{K} \beta_{j}-\rho_{o p} \sigma_{o p, k}^{2}+1} \\
&=-(M+1)+\frac{(M+1)}{1-\lambda_{o p, k}}
\end{aligned}
$$

where $\lambda_{o p, k}=\frac{\beta_{k}^{2}}{\left(\frac{1}{\rho_{o p}}+\sum_{j=1}^{K} \beta_{j}\right)\left(\frac{1}{\rho_{o p}}+\beta_{k}\right)}$. Furthermore, based on (22) and the fact that $\lim _{\rho_{p p} \rightarrow \infty} \varepsilon_{t, i}^{2}=0$ for any $i$ and $t$, we have

$$
\lim _{\rho_{p p} \rightarrow \infty}\left(\frac{\sigma_{\mathbf{n}^{k}[i]}^{2}}{\rho_{p p}}+\beta_{k}\right)=\beta_{k} .
$$

It is easily seen that $\lim _{\rho_{p p} \rightarrow \infty} \lambda_{p p, k}=\lim _{\rho_{p p} \rightarrow \infty} \lambda_{o p, k}=\frac{\beta_{k}}{\sum_{j=1}^{K} \beta_{j}}$.

Further, it achieves that $\lim _{\rho_{p p} \rightarrow \infty} \gamma_{k, i}^{u}=\lim _{\rho_{p p} \rightarrow \infty} \gamma_{o p, k}^{u}$. Define $\bar{R}_{k, o p}^{u}=\lim _{\rho_{p p} \rightarrow \infty} R_{o p, k}^{u}$ and $\bar{R}_{k, p p}^{u}=\lim _{\rho_{p p} \rightarrow \infty} R_{k, i}^{u}$. We have

$$
\bar{R}_{k, p p}^{u}=\bar{R}_{k, o p}^{u} .
$$

The similar manipulations to (49) is applied to $R_{k, 1}^{u_{k_{1}}}$ and $R_{k, i}^{u_{k_{1}}}$ in (41) and (42), respectively, and it achieves that $\lim _{\rho_{p p} \rightarrow \infty} R_{k, 1}^{u_{k_{1}}} \geq \lim _{\rho_{p p} \rightarrow \infty} R_{k, i}^{u_{k_{1}}}=\bar{R}_{k, p p}^{u}$.

Then considering the value of $\tilde{R}_{p p}^{d}$ at asymptotically high SNRs, we first compute the downlink rate $\gamma_{k, i}^{d}$ in (33). Following the steps in (49), it achieves

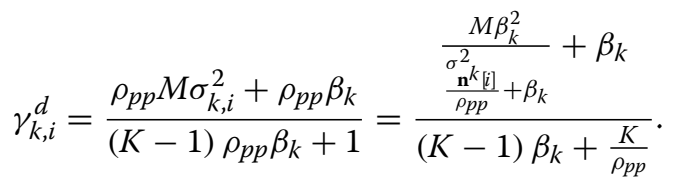

Applying the similar manipulations in (48), it arrives at

$$
\gamma_{o p, k}^{d}=\frac{\frac{M \beta_{k}^{2}}{\frac{1}{\rho_{o p}}+\beta_{k}}+\beta_{k}}{(K-1) \beta_{k}+\frac{K}{\rho_{o p}}} .
$$

Therefore, based on (51), it is easily seen that $\lim _{\rho_{p p} \rightarrow \infty} \gamma_{k, i}^{d}=\lim _{\rho_{p p} \rightarrow \infty} \gamma_{o p, k}^{d}$. Define $\bar{R}_{k, p p}^{d}=\lim _{\rho_{p p} \rightarrow \infty} R_{k, i}^{d}$ and $\bar{R}_{k, o p}^{d}=\lim _{\rho_{p p} \rightarrow \infty} R_{o p, k}^{d}$, then we have $\bar{R}_{k, p p}^{d}=\bar{R}_{k, o p}^{d}$.

Thus far, the uplink rates of both pilot designs at high SNR are

$$
\lim _{\rho_{p p} \rightarrow \infty} \tilde{R}_{p p}^{u} \geq \frac{1}{N_{c} T} \sum_{k=1}^{K}\left[\left(T-D_{p p}-1\right) N_{c}-k+1\right] \bar{R}_{k, p p}^{u} .
$$

$$
\lim _{\rho_{p p} \rightarrow \infty} \tilde{R}_{o p}^{u}=\frac{1}{N_{c} T} \sum_{k=1}^{K}\left[\left(T-D_{o p}-K\right) N_{c}\right] \bar{R}_{k, o p}^{u} .
$$

And the downlink rates of both pilot designs at high SNR are

$$
\lim _{\rho_{p p} \rightarrow \infty} \tilde{R}_{p p}^{d}=\frac{1}{N_{c} T} \sum_{k=1}^{K} D_{p p} N_{c} \bar{R}_{k, p p}^{d} .
$$




$$
\lim _{\rho_{p p} \rightarrow \infty} \tilde{R}_{o p}^{d}=\frac{1}{N_{c} T} \sum_{k=1}^{K} D_{o p} N_{c} \bar{R}_{k, o p}^{d} .
$$

Some remarks on the high SNR analysis show the priority of our proposed pilot design over the conventional one.

\subsection{Low SNR analysis}

In the sequel, consider the performance of two pilot designs at an asymptotically low SNR. Following the steps in (49) and based on the fact that $\sigma_{\mathbf{n}^{k}[i]}^{2} \leq 1+\rho_{p p} \sum_{j=1, j \neq k}^{K} \beta_{j}$, we have

$$
\gamma_{k, i}^{u} \geq-(M+1)+\frac{(M+1)}{1-\underline{\lambda}_{p p, k}}
$$

where $\underline{\lambda}_{p p, k}=\frac{\beta_{k}^{2}}{\left(\frac{1}{\rho_{p p}}+\sum_{j=1}^{K} \beta_{j}\right)^{2}}$. Furthermore, based on $\rho_{p p}=$ $\frac{2 T N_{c}}{2 T N_{c}-K+1} \rho_{o p}$, we have

$$
\frac{1}{\rho_{o p}}+\beta_{k}=\frac{1}{\rho_{p p}}+\sum_{j=1}^{K} \beta_{j}-\sum_{j=1, j \neq k}^{K} \beta_{j}+\frac{K-1}{\left(2 T N_{c}-K+1\right) \rho_{p p}},
$$

where the last term tends to infinity at asymptotically low SNR. Compared with $\lambda_{o p, k}$, it arrives at the result that $\lim _{\rho_{p p} \rightarrow 0} \gamma_{k, i}^{u} \geq \lim _{\rho_{p p} \rightarrow 0} \gamma_{o p, k}^{u}$, which is also applied to $\gamma_{k, 1}^{u_{k_{1}}}$ and $\gamma_{k, i}^{u_{k_{1}}}$ in (41) and (42). Define $\underline{R}_{k, o p}^{u}=\lim _{\rho_{p p} \rightarrow \infty} R_{o p, k}^{u}$ and $\underline{R}_{k, p p}^{u}=\min \lim _{\rho_{p p} \rightarrow 0}\left\{R_{k, i}^{u}, R_{k, 1}^{u_{k_{1}}}, R_{k, i}^{u_{k_{1}}}\right\}$, then we have $\underline{R}_{k, p p}^{u} \geq$ $\underline{R}_{k, o p}^{u}$.

As for the value of $\tilde{R}_{p p}^{d}$ at asymptotically low SNR, it arrives at

$$
\gamma_{k, i}^{d} \geq \frac{\frac{M \beta_{k}^{2}}{\frac{1}{\rho_{p p}}+\sum_{j=1}^{K} \beta_{j}}+\beta_{k}}{(K-1) \beta_{k}+\frac{K}{\rho_{p p}}} .
$$

Define $\underline{R}_{k, p p}^{d}=\lim _{\rho_{p p} \rightarrow 0} R_{k, i}^{d}$ and $\underline{R}_{k, p p}^{d}=\lim _{\rho_{o p} \rightarrow 0} R_{o p, k}^{d}$, then obviously, we have $\underline{R}_{k, p p}^{d} \geq \underline{R}_{k, o p}^{d}$.

Above all, the uplink rates of both pilot designs at low SNR are

$$
\lim _{\rho_{p p} \rightarrow 0} \tilde{R}_{p p}^{u} \geq \frac{1}{N_{c} T} \sum_{k=1}^{K}\left[\left(T-D_{p p}-1\right) N_{c}-k+1\right] \bar{R}_{k, p p}^{u} .
$$

$$
\lim _{\rho_{p p} \rightarrow 0} \tilde{R}_{o p}^{u}=\frac{1}{N_{c} T} \sum_{k=1}^{K}\left[\left(T-D_{o p}-K\right) N_{c}\right] \underline{R}_{k, o p}^{u}
$$

And the downlink rates of both pilot designs at low SNR are

$$
\begin{aligned}
& \lim _{\rho_{p p} \rightarrow 0} \tilde{R}_{p p}^{d}=\frac{1}{N_{c} T} \sum_{k=1}^{K} D_{p p} N_{c} \underline{R}_{k, p p}^{d} . \\
& \lim _{\rho_{p p} \rightarrow 0} \tilde{R}_{o p}^{d}=\frac{1}{N_{c} T} \sum_{k=1}^{K} D_{o p} N_{c} \underline{R}_{k, o p}^{d} .
\end{aligned}
$$

Fortunately, based on the above analysis, we are able to achieve Theorem 1 characterizing the advantage of our proposed pilot design.

Theorem 1. The proposed pilot design outperforms the conventional one for both low and high SNRs.

Proof. Assume that $D_{p p}=D_{o p}$. Based on (55) to (58), we have

$$
\lim _{\rho_{p p} \rightarrow \infty}\left(\tilde{R}_{p p}^{u}-\tilde{R}_{o p}^{u}\right)>\frac{1}{N_{c} T} \sum_{k=1}^{K}\left[(K-1) N_{c}-k+1\right] \bar{R}_{k, p p}^{u}
$$

and

$$
\lim _{\rho_{p p} \rightarrow \infty}\left(\tilde{R}_{p p}^{d}-\tilde{R}_{o p}^{d}\right)=0 .
$$

Therefore, it arrives at

$$
\lim _{\rho_{p p} \rightarrow \infty}\left(C_{p p}^{s}-C_{o p}^{s}\right) \geq 0 .
$$

The similar result at asymptotically low SNR can be achieved by using (62) to (65):

$$
\lim _{\rho_{p p} \rightarrow 0}\left(C_{p p}^{s}-C_{o p}^{s}\right) \geq 0 .
$$

The conclusion in Theorem 1 is independent of coherence interval length $T$ and number of concerned coherence intervals $N_{c}$. It provides a superior pilot design for scenarios of small or large noise and interference.

\section{Numerical results}

In this section, we present some numerical results about the performance of the proposed pilot design. The system tested here consists of $K=5$ users within the same distance from the BS. Without loss of generality, assume that the large-scale fading coefficients $\beta_{k}$ are all 1 . In practice, the users can be scheduled according to their channel conditions. If it is not specified, the number of $\mathrm{BS}$ antennas is set to be 128 , and $\rho_{u}=\rho_{d}=\rho_{p}$.

First, we compare the system overall achievable rate of our proposed pilot design with the conventional one under different numbers of concerned coherence intervals with varying SNR. Figure 3 shows that, with the proposed pilot design, the system overall achievable rates 


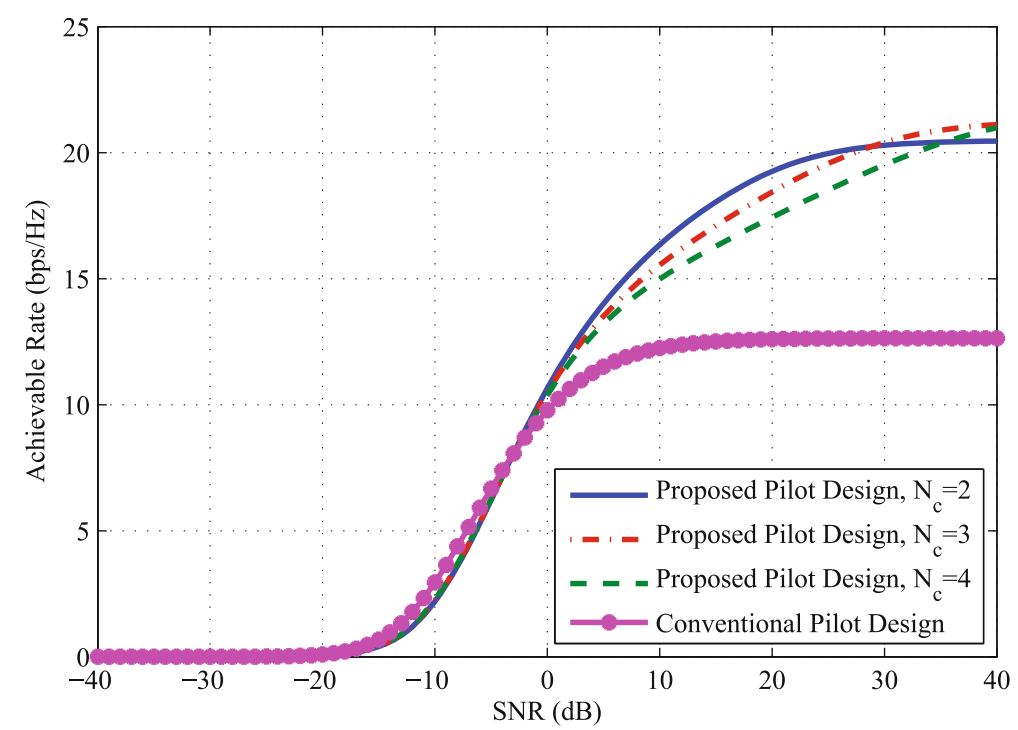

Figure 3 Comparison of system overall achievable rates of two designs versus SNR. We set $T=10$ and $D_{o p}=D_{p p}=3$.

with different $N_{c}$ all surpass the rate of the conventional one when SNR varies from $-1 \mathrm{~dB}$ to $40 \mathrm{~dB}$, which verifies Theorem 1. Further, the system overall achievable rate keeps increasing along with SNR when SNR is high, whereas that of the conventional pilot design tends to saturate. Besides, a larger $N_{c}$ generally leads to a smaller system overall achievable rate due to the accumulative CSI estimation error. However, $N_{c}$ impacts little on the comparison of system overall achievable rates between two pilot designs.

Then, we consider the performance of two pilot designs with various number of BS antennas. Figure 4 shows that the system overall achievable rates of both pilot designs improve as the number of BS antennas grows.
This results from the fact that the channel vectors tend to be orthogonal as the number of BS antennas increases. Moreover, the system overall achievable rates of the proposed pilot design respectively outperform those of the conventional one.

Moreover, Figure 5 illustrates that the system overall achievable rates of our proposed pilot design achieve a more significant performance gain compared with those of the conventional one as the number of users increases. This verifies again the priority of our proposed pilot design when the number of users is large.

According to Figures 3, 4, and 5, we conclude that the proposed pilot design provides a performance improvement compared with the conventional one at most SNR

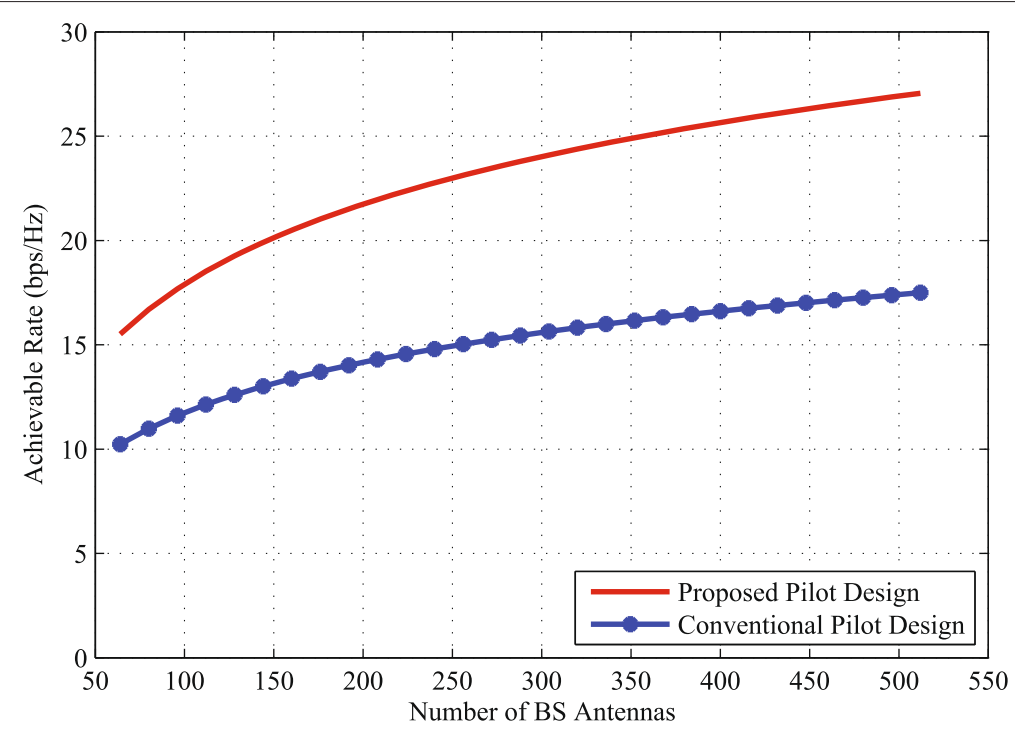

Figure 4 Comparison of system overall achievable rates of two designs versus number of $\mathbf{B S}$ antennas. We set $T=20$ and $N_{c}=2$. 


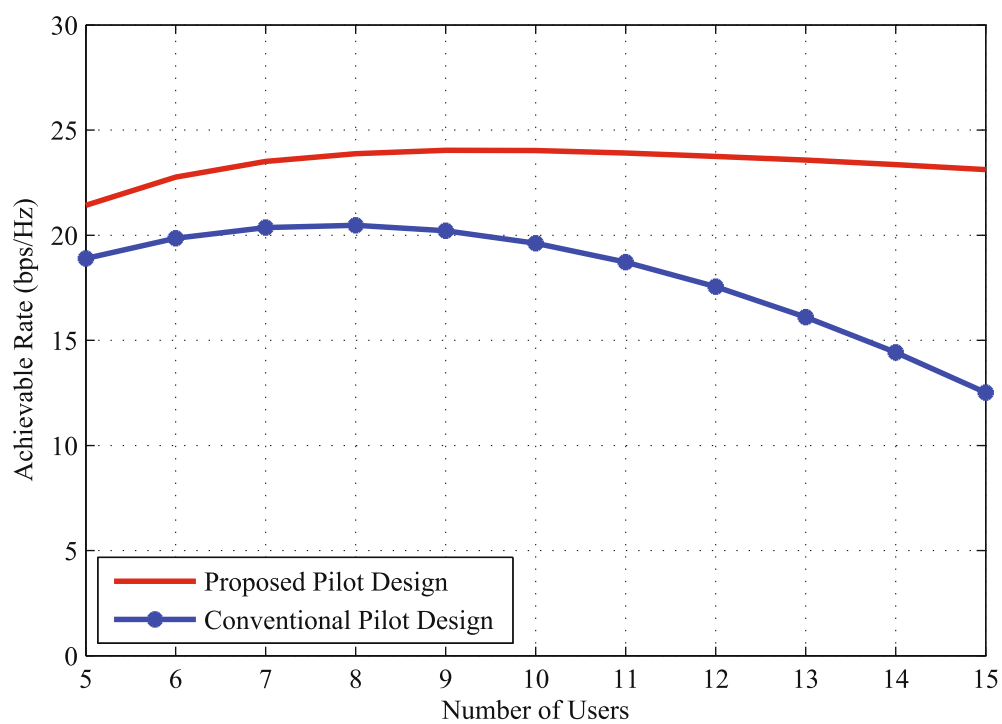

Figure 5 Comparison of system overall achievable rates of two designs versus number of users. We set $T=20$ and $N_{C}=2$.

under the above scenario due to the tradeoff between pilot consumption and channel estimation accuracy.

Further, in Figure 6, we compare two pilot designs considering SNR and the length of coherence interval $T$. We assume that the length of downlink data per coherence interval is $\left\lfloor\frac{3(T-K)}{4}\right\rfloor$, where $\lfloor\cdot\rfloor$ means rounding down to the closest integer. Figure 6 shows that the proposed pilot design outperforms the conventional one at low and high SNRs no matter how long the coherence interval is, which also coincides with Theorem 1. Besides, the shorter the coherence interval is, the larger the SNR region is, in which the system overall achievable rates of the proposed pilot design win. This again strengthens the motivation and effectiveness of our proposed pilot design.

Generally, massive MIMO in many cases works at low SNRs. We have analyzed the performance at low SNRs in both theoretical and numerical ways. Theorem 1 indicates that our proposed pilot design outperforms the conventional one at low SNRs, which is later validated by simulation results in Figure 6.

Concerning the high SNR analysis in both Theorem 1 and Figures 3 and 6, this is due to the consideration that massive MIMO applies not only for future wireless communication systems but also for current long

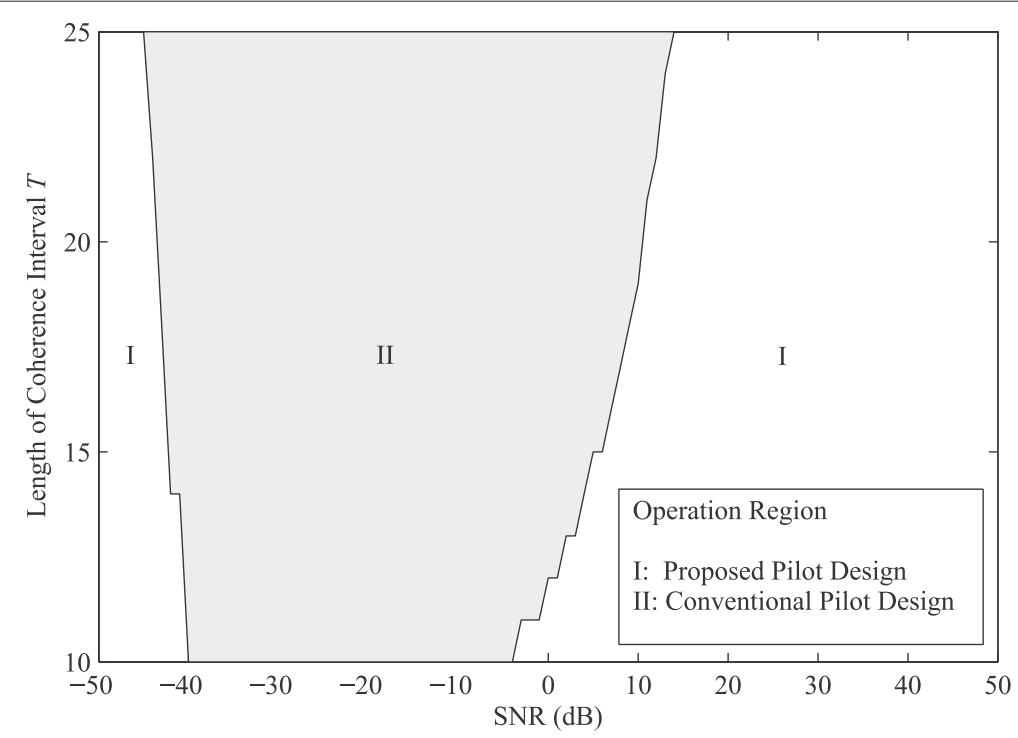

Figure 6 Comparison of system overall achievable rates of two designs considering SNR and $T$. We set $N_{C}=2$. 
term evolution/long term evolution-advanced (LTE/LTEA) systems. For systems like LTE/LTE-A, the operation region in terms of SNR varies widely, for example, from $3 \mathrm{~dB}$ to $30 \mathrm{~dB}$ [20]. In particular, for users locating in the proximity of BS, they experience a relatively high quality of SNR. Moreover, users are more likely to experience high SNR transmissions especially for the emerging small cell deployment with reduced cell sizes. The combination of small cells and massive MIMO [21] could lead to a high SNR scenario. In Theorem 1, the high SNR analysis validates the application of our proposed pilot design for these scenarios. Finally, the analysis for both low and high SNRs presents a complete performance comparison between two pilot designs.

\section{Conclusions}

This paper proposes a semi-orthogonal pilot design using SIC in a TDD massive MIMO system, which makes full use of the asymptotic channel orthogonality. The performance of the proposed pilot design is elaborated both theoretically and numerically. Simulation results show that the proposed pilot design outperforms the conventional orthogonal pilots. And particularly for low or high SNRs with any coherence interval length, the superiority of our proposed pilot design is theoretically proven.

Competing interests

The authors declare that they have no competing interests.

\section{Acknowledgements}

Part of this paper will be presented at the IEEE Globecom, Austin, USA, Dec. 2014 [22]. This work was supported by the 973 Program under 2013CB329203, the NSFC under 61471114 and 61223001, and the Important National Science \& Technology Specific Projects $2012 Z$ Z03001038 and 2013ZX03003016.

Received: 7 August 2014 Accepted: 24 November 2014

Published: 11 December 2014

\section{References}

1. EG Larsson, F Tufvesson, O Edfors, TL Marzetta, Massive MIMO for next generation wireless systems. IEEE Commun. 52(2), 186-195 (2014)

2. TL Marzetta, Noncooperative cellular wireless with unlimited numbers of base station antennas. IEEE Trans. Wireless Commun. 9(11), 3590-3600 (2010)

3. J Hoydis, S ten Brink, M Debbah, Massive MIMO in the UL/DL of cellular networks: How many antennas do we need? IEEE J. Select. Areas Commun. 31(2), 160-171 (2013)

4. HQ Ngo, EG Larsson, TL Marzetta, in Proc. Annual Allerton Conference on Communication, Control, and Computing (Allerton), Massive MU-MIMO downlink TDD systems with linear precoding and downlink pilots, (Monticello, IL, 2013), pp. 293-298

5. J Jose, A Ashikhmin, TL Marzetta, S Vishwanath, in Proc. IEEE International Symposium on Information Theory (ISIT), Pilot contamination problem in multi-cell TDD systems, (Seoul, Korea, 2009), pp. 2184-2188

6. Z Xiang, M Tao, X Wang, Massive MIMO multicasting in noncooperative cellular networks. IEEE J. Select. Areas Commun. 32(6), 1180-1193 (2014)

7. K Guo, Y Guo, G Fodor, G Ascheid, in Proc. IEEE ICC, Uplink power control with MMSE receiver in multi-cell MU-massive-MIMO systems, (Sydney, Australia, 2014)

8. J Jose, A Ashikhmin, TL Marzetta, S Vishwanath, in IEEE Trans. Wireless Commun, Pilot contamination and precoding in multi-cell TDD systems, vol. 10, (2011), pp. 2640-2651
9. H Yin, D Gesbert, M Filippou, Y Liu, A coordinated approach to channe estimation in large-scale multiple-antenna systems. IEEE J. Select. Areas Commun. 31(2), 264-273 (2013)

10. F Fernandes, A Ashikhmin, TL Marzetta, Inter-cell interference in noncooperative TDD large scale antenna systems. IEEE J. Select. Areas Commun. 31(2), 192-201 (2013)

11. A Ashikhmin, T Marzetta, in Proc. IEEE ISIT, Pilot contamination precoding in multi-cell large scale antenna systems, (Cambridge, USA, 2012), pp. 1137-1141

12. A Hu, T Lv, H Gao, Y Lu, E Liu, in Proc. IEEE ICC, Pilot design for large-scale multi-cell multiuser MIMO systems, (Budapest, Hungary, 2013), pp. 5381-5385

13. F Rusek, D Persson, BK Lau, EG Larsson, TL Marzetta, O Edfors, F Tufvesson, Scaling up MIMO: Opportunities and challenges with very large arrays. IEEE Sig. Proc. Mag. 30(1), 40-60 (2013)

14. X Liu, S Lu, ME Bialkowski, HT Hui, in Proc. Asia-Pacific Microwave Conference (APMC), MMSE channel estimation for MIMO system with receiver equipped with a circular array antenna, (Bangkok, Thailand, 2007), pp. 1-4

15. SM Kay, Fundamentals of Statistical Signal Processing: Theory (Prentice-Hall, Englewood Cliffs, NJ, 2013)

16. HQ Ngo, EG Larsson, TL Marzetta, Energy and spectral efficiency of very large multiuser MIMO systems. IEEE Trans. Wireless Commun. 61(4), 1436-1449 (2013)

17. H Yang, TL Marzetta, Performance of conjugate and zero-forcing beamforming in large-scale antenna systems. IEEE J. Select. Areas Commun. 31(2), 172-179 (2013)

18. XWu, W Xu, in Proc. IEEE WCNC, Downlink performance analysis with enhanced multiplexing gain in multi-cell large-scale MIMO under pilot contamination, (Istanbul, Turkey, 2014)

19. Al Khuri, Advanced Calculus With Applications In Statistics (John Wiley and Sons, New York, 2003)

20. Evolved universal terrestrial radio access (E-UTRA); user equipment (UE) radio transmission and reception (release 10). 3GPP TS 36.101 V10.3.0 (2011)

21. MK Emil Bjornson, M Debbah, in Proc. ICT, Massive MIMO and small cells: improving energy efficiency by optimal soft-cell coordination, (Casablanca, Morocco, 2013)

22. $X$ Zheng, $H$ Zhang, $W X u, X Y$ You, in Proc. IEEE Globecom, Semi-orthogonal pilot design for massive MIMO systems using successive interference cancellation, (Austin, USA, 2014)

doi:10.1186/1687-1499-2014-220

Cite this article as: Zhang et al:: On massive MIMO performance with semi-orthogonal pilot-assisted channel estimation. EURASIP Journal on Wireless Communications and Networking 2014 2014:220.

\section{Submit your manuscript to a SpringerOpen ${ }^{\circ}$ journal and benefit from:}

- Convenient online submission

- Rigorous peer review

- Immediate publication on acceptance

- Open access: articles freely available online

- High visibility within the field

- Retaining the copyright to your article

Submit your next manuscript at $>$ springeropen.com 